\title{
Temozolomide induces autophagy in primary and established glioblastoma cells in an EGFR independent manner
}

\author{
SILVIA WÜRSTLE ${ }^{1}$, FABIAN SCHNEIDER ${ }^{1}$, FLORIAN RINGEL ${ }^{2,3}$, JENS GEMPT ${ }^{2}$, \\ FRIEDERIKE LÄMMER $^{1}$, CLAIRE DELBRIDGE ${ }^{1}$, WEI WU ${ }^{1}$ and JÜRGEN SCHLEGEL ${ }^{1}$ \\ Departments of ${ }^{1}$ Neuropathology and ${ }^{2}$ Neurosurgery, Technische Universität München, D-81675 Munich; \\ ${ }^{3}$ Department of Neurosurgery, Universitätsmedizin Mainz, D-55131 Mainz, Germany
}

Received September 2, 2016; Accepted January 13, 2017

DOI: 10.3892/ol.2017.6107

\begin{abstract}
Despite major contributions to the current molecular understanding of autophagy, a recycling process for intracellular components to maintain homeostatic balance, relatively little is known about the interacting networks. To address this issue, the current study investigated the role of autophagy in primary and established glioblastoma multiforme (GBM) cells and its interplay with the epidermal growth factor receptor (EGFR) and the standard chemotherapeutic agent temozolomide (TMZ). TMZ treatment leads to an upregulation of autophagy, predominantly in primary GBM cells. The interaction between EGFR and Beclin-1, an important protein in initiating autophagy, was assessed using a cancer cell line transfected with EGFR ${ }^{\text {VIII }}$, and by stimulation with EGF. The results of the current study suggest that Beclin-1 and EGFR do not interact directly in either primary or established GBM cells. To enable the limited efficacy of patient treatment strategies of GBM to potentially be enhanced through the application of autophagy regulators, the multiple cellular interactions of autophagy require further elucidation.
\end{abstract}

\section{Introduction}

Glioblastoma multiforme (GBM) is not only the most common type of brain tumor in adulthood, but also one of the most malignant types of cancer, with a median survival time (World Health Organization grade IV) of 14-15 months with

Correspondence to: Ms. Silvia Würstle, Department of Neuropathology, Technische Universität München, Trogerstraße 18, D-81675 Munich, Germany

E-mail: silviawuerstle@web.de

Abbreviations: Atg, autophagy-related gene; GBM, glioblastoma multiforme; LC3, microtubule-associated protein light chain 3; pGBM, primary glioblastoma multiforme cell line; TMZ, temozolomide

Key words: autophagy, epidermal growth factor receptor, temozolomide maximum treatment (1). Its infiltrative and heterogeneous characteristics, combined with high proliferation rates, makes treatment challenging. Standard therapy includes surgery, adjuvant radiotherapy and chemotherapy with the alkylating agent temozolomide (TMZ) (1). TMZ was first identified by Stevens et al in 1984 (2) as an oral anti-cancer treatment. The active metabolite of TMZ methylates guanine residues in the DNA, leading to double strand breaks (3). The O6-methylguanine-DNA methyltransferase (MGMT) gene codes for a protein that removes the methylation performed by TMZ and abrogates its effects (4). This gene's promoter is methylated in $\sim 50 \%$ of cases, which is an independent and favorable prognostic factor for patients with GBM (4).

Previous studies have established the association between chemotherapy with TMZ and autophagy induction $(5,6)$. Autophagy, from the Greek meaning 'self-eating', is a recycling machinery of intracellular proteins and organelles like mitochondria (7). Macroautophagy is the major type of autophagy and is referred to as autophagy for the remainder of the current study. This process comprises several sequestration steps, beginning with a phagophore enclosing cellular waste and forming the autophagosome by elongation (8). Fusion of the autophagosome and a lysosome allows acidic hydrolases to degrade the inner components of the now termed 'autolysosome' (8).

For the purposes of monitoring the autophagic flux, the microtubule-associated protein light chain 3 (LC3) is one of the most reliable proteins (8). Pro-LC3 is cleaved by the autophagy-related-gene protease 4 (Atg4) to form LC3-I (9). The conjugation of LC3-I to phosphatidylethanolamine forms LC3-II, which is the lipidated form of LC3 and is located at the autophagosome cytosolic and intraluminal membrane (8). Subsequently,LC3-II at the intraluminal membrane is degraded by lysosomal hydrolyases in the final step of autophagy (8). The drug chloroquine inhibits this last step, resulting in an accumulation of LC3-II (10).

Beclin-1, the mammalian homolog of Atg6, is required to initiate the autophagic process $(11,12)$. Notably, Wei et al $(13)$ described an interaction between the epidermal growth factor receptor (EGFR) and autophagy in non-small cell lung cancer (NSCLC) cells. Active EGFR inhibited the initiation of autophagy via phosphorylation of Beclin-1, and activation of EGFR was performed through the addition of EGF or by 
transfection of cells with the truncated EGFR ${ }^{\mathrm{vIII}}$ version (13). This mutated receptor is common in NSCLC cells, as well as in GBM $(14,15)$. Due to an in-frame deletion of exon 2-7, the extracellular binding portion is deleted, leading to the continuous activation of EGFR (15). Wei et al (13) identified that this activation inhibits autophagy through an interaction with Beclin-1 (13). It remains to be established whether the same underlying mechanisms are relevant in GBM.

\section{Materials and methods}

Reagents. TMZ and chloroquine were obtained from Sigma-Aldrich (Merck KGaA, Darmstadt, Germany). EGF was purchased from PeproTech Inc. (Rocky Hill, NJ, USA).

Cell culture and cell treatment. The LN18 human glioblastoma cell line, first characterized in 1981 (16), was obtained from Dr. Van Meir (University of Lausanne, Switzerland). pGBM T1 and pGBM T12 cells were isolated from tissues that were collected in cooperation with the Department for Neurosurgery at Technische Universität München (Munich, Germany). Primary cell culture was established by Dr. Andrea Schäfer as previously described (17). The resection (January and May 2008) and subsequent processing were performed with patients' consent according to the Technische Universität München medical faculty's guidelines for tissue preservation. Primary single cell suspensions were only cultured at early passages. The primary glioblastoma stem cell line X01 was obtained from Dr Andreas Androutsellis-Theotokis (Carl Gustav Carus University, Dresden, Germany).

LN18, pGBM T1 and pGBM T12 cells were maintained in Dulbecco's modified Eagle's medium (Thermo Fisher Scientific, Inc., Waltham, MA, USA) under standard cell culture conditions in the presence of $5 \% \mathrm{CO}_{2}$ at $37^{\circ} \mathrm{C}$ in a humidified incubator. When treating cells with chemotherapeutics, the concentration of fetal calf serum (FCS; Thermo Fisher Scientific, Inc.) was reduced from 4 to $0.1 \%$ to minimize off-target FCS effects. The primary glioblastoma tumor stem cell line X01 was cultured in RPMI-1640 medium (Thermo Fisher Scientific, Inc.) supplemented with $1 \%$ L-glutamine (Thermo Fisher Scientific, Inc.), 1\% N1 (Thermo Fisher Scientific, Inc.), $1 \%$ non-essential amino acids (Thermo Fisher Scientific, Inc.), $1 \mathrm{ng} / \mathrm{ml}$ transforming growth factor $\beta$ (Thermo Fisher Scientific, Inc.), $20 \mathrm{ng} / \mathrm{ml}$ basic fibroblast growth factor (Thermo Fisher Scientific, Inc.), 20\% BIT100 (Pelo Biotech $\mathrm{GmbH}$, Planegg, Germany), $0.1 \%$ primocin (InvivoGen, San Diego, USA), and $20 \mathrm{ng} / \mathrm{ml}$ epidermal growth factor (PeproTech, Inc.) to simulate cancer stem cell conditions. To investigate EGFR alterations, LN18 cells were transfected with plasmid DNA carrying the constitutively active EGFR ${ }^{\mathrm{VIII}}$ variant $\left(\mathrm{LN} 18^{\mathrm{vIII}}\right)$ by using lipofectamine transfection reagent (Thermo Fisher Scientific, Inc.). Cells were cultured and maintained in the presence of the selective antibiotic G418 (Thermo Fisher Scientific, Inc.). Stable expression of EGFR ${ }^{\mathrm{vIII}}$ was routinely analyzed by western blotting using EGFR antibody (Ab)-12 (cocktail R19/48; 1:500; catalog no. MS-400-P; Thermo Fisher Scientific, Inc.). The antibody is able to detect extracellular and cytoplasmic domains allowing differentiation between wild-type EGFR (170 kDa) and EGFR ${ }^{\mathrm{vIII}}(145 \mathrm{kDa})$ forms of the protein.
LN18, pGBM T1 and pGBM T12 were exposed to $50 \mu \mathrm{M}$ chloroquine for $2 \mathrm{~h}$. LN18 cells were treated with $20 \mathrm{ng}$ EGF for $30 \mathrm{~min}$. The concentration of TMZ treatment varied from 100-500 $\mu \mathrm{M}$ for 2-72 $\mathrm{h}$ due to different responses in established and primary cell lines.

Hypoxic treatment of cells. pGMB X01 cells were placed in the hypoxic incubating chamber at $37^{\circ} \mathrm{C}$ and $\mathrm{O}_{2}$ was gradually replaced by nitrogen within 11 cycles. Following incubation for $24 \mathrm{~h}$ at $1 \% \mathrm{O}_{2}$, the cells were lysed at the same time as the normoxic pGBM X01 control cells.

Immunoblotting. Collected and washed LN18, LN18 pGBM T1 and pGBM T12 proteins were resuspended in freshly prepared lysis buffer (20\% L-Buffer, 2\% phenylmethanesulfonyl fluoride; Cell Signaling Technology, Inc., Danvers, MA, USA) and incubated rotating at $4^{\circ} \mathrm{C}$ for $10 \mathrm{~min}$. Protein quantification was measured using a Bradford protein assay (Bio-Rad Laboratories, Inc., Hercules, CA, USA). Following quantification, equal amounts of proteins $(20 \mu \mathrm{g})$ were unfolded and charged by the detergent sodium dodecyl sulfate. SDS-PAGE gels were prepared with the permeability (7-12\%) adjusted to the size of the proteins. Following separation through the gel matrix, proteins were transferred to an immobilizing polyvinylidene difluoride membrane. Blocking of nonspecific binding sites was performed with $5 \%$ milk for $1 \mathrm{~h}$ at room temperature. Cells were incubated with the following primary antibodies by rotating at $4^{\circ} \mathrm{C}$ overnight: Beclin-1 (1:1,000; catalog no. 4445S; Cell Signaling Technology, Inc., Danvers, MA, USA), light chain 3 B-II (LC3B; 1:1,000; catalog no. 4445S; Cell Signaling Technology, Inc.), MGMT (1:1,000; catalog no. 2739; Cell Signaling Technology, Inc.), EGFR Ab-12 cocktail R19/48 (1:500; catalog no. MS-400-P; Thermo Fisher Scientific, Inc.) and glyceraldehyde-3-phosphate dehydrogenase (GAPDH) (1:10,000; catalog no. G8795; Sigma-Aldrich, Merck KGaA). Blots were incubated with horseradish peroxidase-conjugated anti-mouse or anti-rabbit secondary immunoglobulin for $1 \mathrm{~h}$ at room temperature $(1: 10,000$; catalog nos. 7076 and 7074, respectively; Cell Signaling Technology, Inc.). Every step was followed by three 5 min washes with phosphate-buffered saline (PBS; Thermo Fisher Scientific, Inc.). To visualize the binding sites, enhanced chemiluminescence solution (Thermo Fisher Scientific, Inc.) was added to the blots, and the chemiluminescence reaction was detected using an X-ray film.

Immunofluorescence. LN18 and LN18 ${ }^{\mathrm{vIII}}$ cells were cultured on gelatin-coated glass slips for $48 \mathrm{~h}$ prior to TMZ $(500 \mu \mathrm{M})$ application. At $48 \mathrm{~h}$ following treatment, cells were fixed with $4 \%$ paraformaldehyde at room temperature for $30 \mathrm{~min}$ and washed 3 times with PBS. To allow access to intracellular antigens, cells were exposed to the surfactant $0.1 \%$ Triton X-100 (Carl Roth GmbH+Co. KG, Karlsruhe, Germany)/PBS for 10 min. Blocking of unspecific binding sites was conducted with an antibody blocking buffer containing $1 \%$ bovine serum albumin (Bio-Rad Laboratories), 0.01\% Tween-20 (Carl Roth $\mathrm{GmbH}+\mathrm{Co} . \mathrm{KG}$ ) and $2.5 \%$ goat-serum (Thermo Fisher Scientific, Inc.) for $30 \mathrm{~min}$ at room temperature. The cells were incubated with the primary antibodies against EGFR Ab-12 cocktail R19/48 (1:200; catalog no. MS-400-P; Thermo Fisher Scientific, Inc.) and Beclin-1 (1:200; catalog no. SC-11427; 
Santa Cruz Biotechnology, Inc., Dallas, TX, USA) for $2 \mathrm{~h}$ at room temperature. Following washing with PBS, the cells were exposed to Alexa-568-conjugated anti-mouse (1:500; catalog no. A-11004) and Alexa-488-conjugated anti-rabbit secondary antibodies (1:500; catalog no. A-11008; Thermo Fisher Scientific, Inc.). The cells were subsequently incubated for $45 \mathrm{~min}$ at room temperature in the dark. The slides were analyzed with the Axio Imager 1 microscope (Zeiss AG, Oberkochen, Germany).

Co-immunoprecipitation. To detect protein-protein interactions, a Pierce Classic immunoprecipitation kit (Thermo Fisher Scientific, Inc.) was used according to the manufacturer's protocol. The cells were lysed with ice-cold immunoprecipitation lysis buffer and purified by using control agarose resin. The flow-through was prepared in order to form the immune complex with the primary antibody overnight. The complex was captured in a spin column containing resin slurry by gently mixing for $1 \mathrm{~h}$. Following three washing steps, the immune complex was eluted by incubation in $2 \mathrm{X}$ non-reducing sample buffer with dithiothreitol $(20 \mathrm{mM})$ and collected by centrifugation at $1,000 \times g$ at $4^{\circ} \mathrm{C}$ for $45 \mathrm{sec}$. The antibodies against Beclin-1 (immobilization, 1:100; immunoblotting, 1:1,000; catalog no. 3495; Cell Signaling Technology, Inc.) and EGFR (immobilization, 1:100; immunoblotting 1:1,000; catalog no. 2232; Cell Signaling Technology, Inc.) were used. A protein complex can be pulled down with one or two antibodies depending on whether only the interaction is a matter of interest, or the expression of the two individual proteins is being examined (18). In this case, Beclin-1 and EGFR are established as being expressed in LN18 cells $(19,20)$. For that reason, co-immunoprecipitation was performed using Beclin-1 alone for pull-down.

Statistical analysis. ImageJ (version 1.51; National Institutes of Health, Bethesda, USA) and Microsoft Excel 2007 (Microsoft Corporation, Redmond, WA, USA) were used for analyzing western blots and immunofluorescence. Relative normalization control values of respective GAPDH lanes were used for normalizing the protein of interest. Statistical analysis of Student's $t$-test and Pearson's correlation analysis was performed with R Studio (version 3.2.3; R Studio, Boston, USA). $\mathrm{P}<0.05$ was considered to indicate a statistically significant difference.

\section{Results}

Chloroquine application increases LC3B-II levels. To assess whether the cell lines in the present study are responsive to autophagy, chloroquine was applied to the established cell line LN18 and the primary lines pGBM T1 and pGBM T12. Fig. 1A and the corresponding analysis (Fig. 1B) indicates the significant increase of LC3B-II levels in chloroquine-treated cells $(\mathrm{P}<0.05)$, suggesting a block of autophagy. Beclin-1 was ubiquitously expressed, but its expression levels were not affected by chloroquine treatment.

TMZ induces autophagy in primary and established glioblastoma cells. LN18 cells were treated with 100-200 $\mu \mathrm{M}$ TMZ for $2 \mathrm{~h}$ each, which did not result in an increase in
LC3B-II protein levels (data not shown). Conversely, TMZ treatment $(500 \mu \mathrm{M})$ for $72 \mathrm{~h}$ resulted in a arked upregulation of LC3B-II (Fig. 1C). To investigate these findings with primary cells, pGBM T1 and pGBM T12 cells were exposed to TMZ $(200 \mu \mathrm{M})$, which resulted in a significant increase of LC3B-II in pGBM T1 cells (Fig. 1D and $\mathrm{E} ; \mathrm{P}=0.0168$ ). The primary cell line pGBM T12 exhibited only a minor modification of LC3B-II levels.

TMZ application decreases MGMT protein levels. Methylation of the promoter of the MGMT gene and its associated lowered protein levels results in a higher susceptibility towards TMZ treatment (21). LN18 cells expressed high levels of MGMT protein (Fig. 2A). By contrast, pGBM T1 and pGBM T12 did not express MGMT protein (data not shown). The high levels of MGMT in LN18 cells decreased following the application of TMZ (Fig. 2A and B, $\mathrm{P}<0.05$ ), indicating a reduction in MGMT protein.

EGFR and EGFR ${ }^{\text {vIII }}$ do not phosphorylate Beclin-1 independently of TMZ or EGF application. In the present study, several techniques were applied to monitor the potential interactions of Beclin-1 and EGFR. Immunoblotting revealed the phosphorylation status of Beclin-1, demonstrating that, in LN18 cells, Beclin-1 was not phosphorylated independently of TMZ application. To minimize the effects of inactive EGFR, LN18 cells transfected with the constitutively active vIII mutant were cultured (Fig. 3A). In addition, LN18 was incubated with EGF (20 ng, 30 min). Beclin-1 phosphorylation status was not divergent from the controls following EGF application or in LN18 ${ }^{\text {vIII }}$. LC3B-II levels were not altered in LN18 by stimulation with EGF (Fig. 3B).

EGFR and EGFR ${ }^{\text {vIII }}$ do not co-immunoprecipitate with Beclin-1 independently of TMZ or EGF application. In the GBM cell lines LN18 and LN18 ${ }^{\text {vIII }}$, Beclin-1 and EGFR did not co-immunoprecipitate (Fig. 3C and D). The EGFR-Beclin-1 interaction was not promoted by treatment with TMZ (500 $\mu \mathrm{M}, 24 \mathrm{~h}$ ) or by stimulation with EGF (20 ng, $30 \mathrm{~min}$; Fig. 3D). These results were further confirmed by co-immunoprecipitation of the primary GBM cell line $\mathrm{X} 01$. To investigate whether hypoxia, as is present in the center of tumor masses, interferes with the EGFR-Beclin-1 association, pGBM X01 was incubated under hypoxic cell culture conditions for $24 \mathrm{~h}$. Formation of the EGFR-Beclin-1 complex was not induced by hypoxia for $24 \mathrm{~h}$ in X01 cells (data not shown).

Immunofluorescence detects varied localizations for EGFR and Beclin-1 independent of TMZ application. Immunofluorescence is a method enabling the visualization of co-localized proteins, suggesting their interaction. As presented in Fig. 4, the majority of EGFR spots could be identified in $\mathrm{LN} 18^{\mathrm{vIII}}$ cells due to the antibody detection of wild-type EGFR, as well as the aberrant form vIII. EGFR and

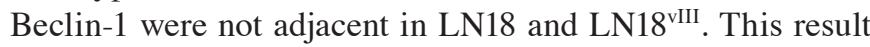
was not altered by treatment with high-dose TMZ. Compared with $\mathrm{LN} 18^{\text {vIII }}$ expressing the truncated and constitutively active EGFR, TMZ treatment appeared to restrict growth more in wild-type LN18 cells. 


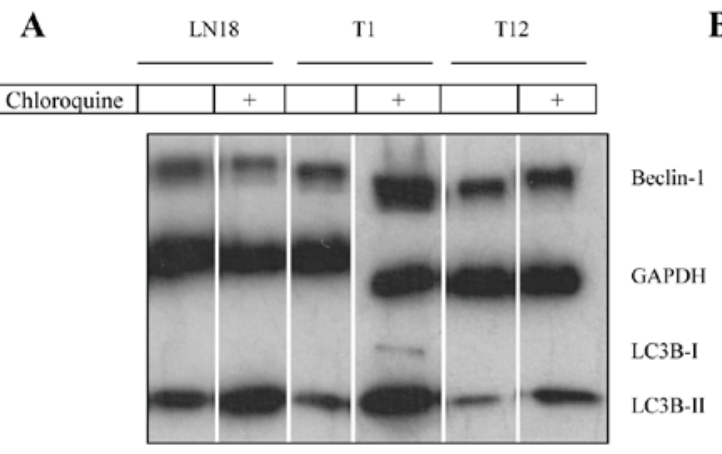

B
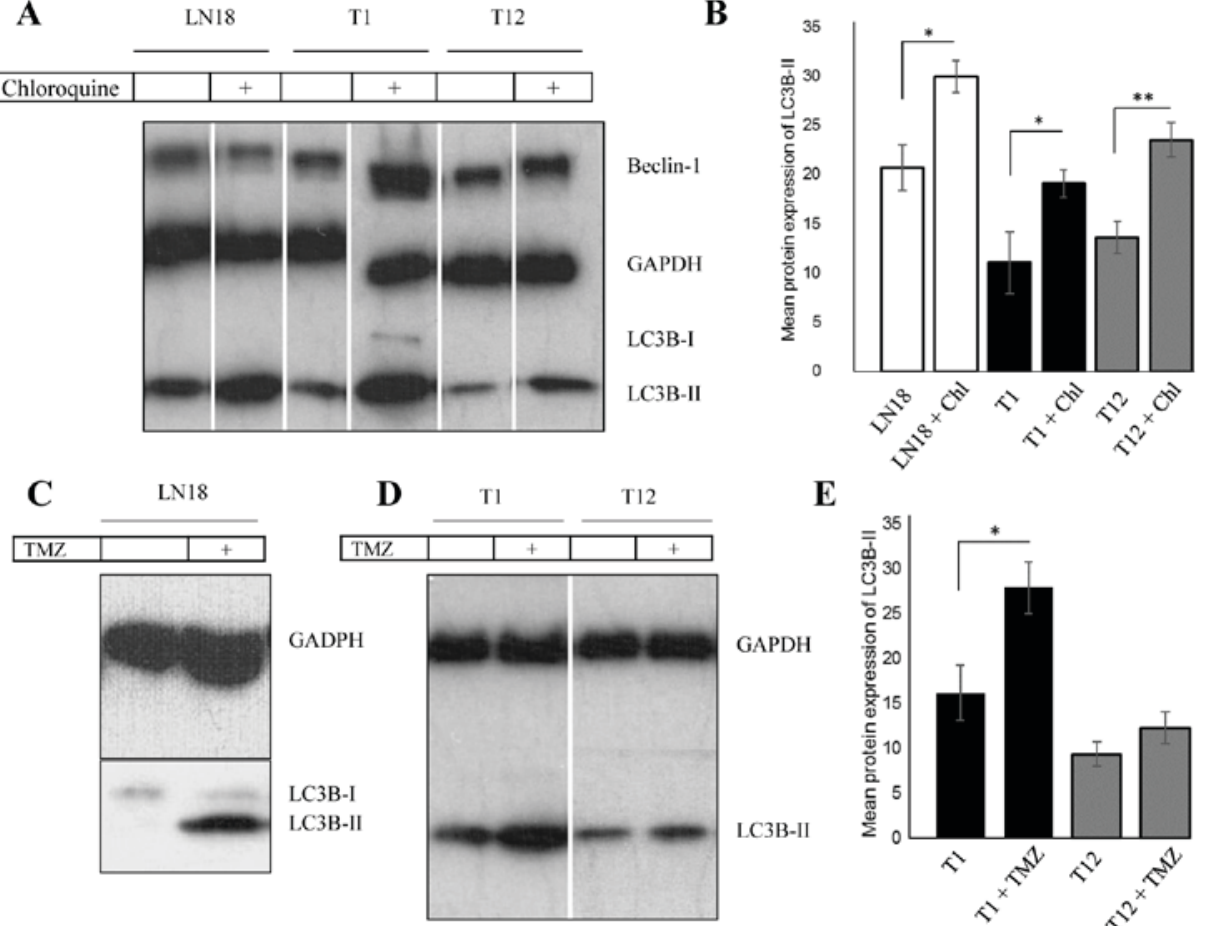

E

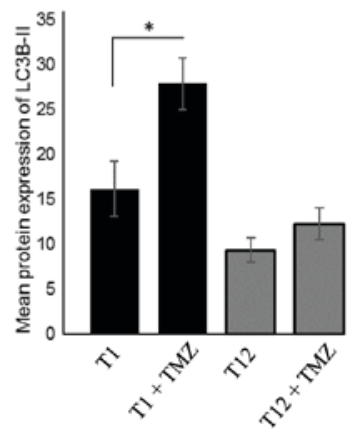

Figure 1. TMZ upregulates LC3B-II levels in primary and established GBM cells. (A) Western blot analysis revealed increased levels of LC3B-II following treatment with $50 \mu \mathrm{M}$ chloroquine for $2 \mathrm{~h}$ in GBM LN18, pGBM T1 and pGBM T12 cells. (B) Analysis of chloroquine treatment, with LC3B-II upregulation normalized to GAPDH. (C) LN18 exhibits an upregulation of LC3B-II following long-term TMZ treatment. TMZ was applied for $72 \mathrm{~h}$ at a concentration of $500 \mu \mathrm{M}$. (D) Autophagy levels were increased in pGBM T1 following short-term application of low-dose TMZ. In pGBM T1 cells, the level of LC3B-II protein was increased following TMZ treatment ( $200 \mu \mathrm{M}, 2 \mathrm{~h})$. In pGBM T12 cells, the LC3B-II protein levels were not notably modified by short-term TMZ treatment. (E) Densitometric analysis of protein expression following TMZ treatment. ${ }^{*} \mathrm{P}<0.05,{ }^{* *} \mathrm{P}<0.01$. Error bars indicate the mean densitometric value \pm standard deviation. Chl, chloroquine; TMZ, temozolomide; LC3B-II, light chain 3 B-II; GAPDH, glyceraldehyde-3-phosphate dehydrogenase; GBM, glioblastoma multiforme; LN18 ${ }^{\text {vIII }}$, constitutively active EGFR ${ }^{\text {vII }}$ variant.

A

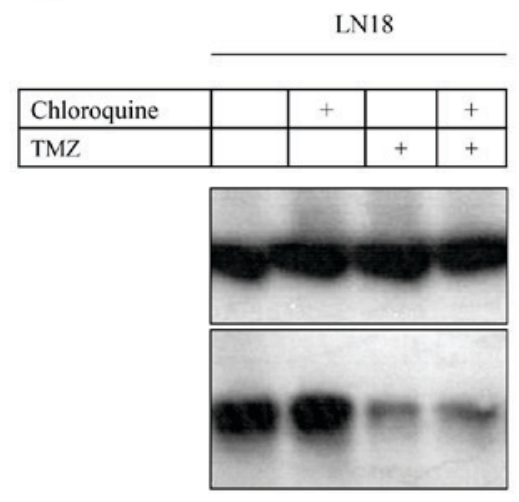

B

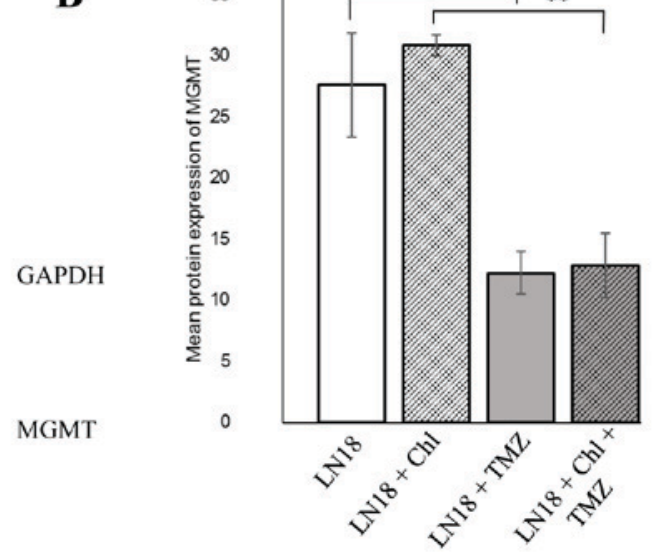

Figure 2. MGMT expression is decreased following TMZ treatment. (A) LN18 cells expressed high levels of MGMT protein, which could be suppressed by TMZ treatment $(200 \mu \mathrm{M}, 24 \mathrm{~h})$. Chloroquine $(50 \mu \mathrm{M}, 2 \mathrm{~h})$ in single or combined treatment had no effect on MGMT expression levels. (B) Densitometric analysis of protein expression following TMZ treatment. ${ }^{*} \mathrm{P}<0.05,{ }^{* *} \mathrm{P}<0.01$. Error bars indicate the mean densitometric value \pm standard deviation. GAPDH, glyceraldehyde-3-phosphate dehydrogenase; MGMT, O6-methylguanine-DNA methyltransferase; Chl, chloroquine; TMZ, temozolomide.

\section{Discussion}

Previous studies on autophagy have provided insight into its underlying mechanisms at a molecular level (22). However, there remain challenges to our understanding, including the investigation of interacting signaling pathways and their impact on the deleterious or protective character of autophagy. With an interdisciplinary effort, the elucidation of the interaction between autophagy proteins and other significant cellular pathways may aid the treatment of malignant cancer, including GBM.

GBM is the most malignant type of brain tumor in adulthood and poses a major challenge in terms of therapy 

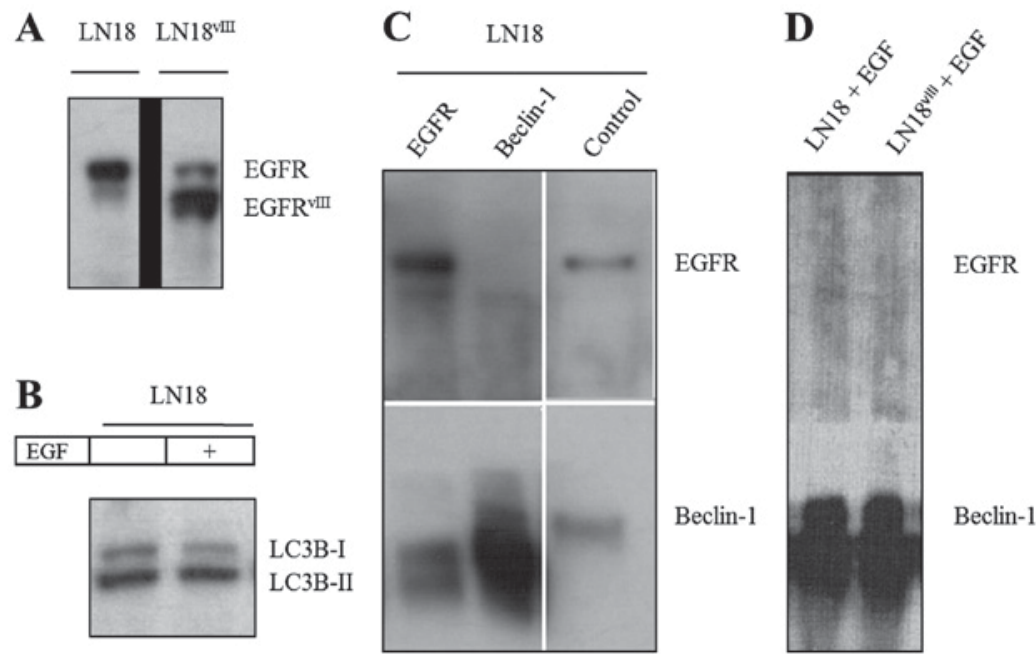

Figure 3. EGFR and Beclin-1 do not interact directly by phosphorylation. (A) Wild-type and truncated EGFR was detected using western blotting. The truncated vIII form was observed at $\sim 145 \mathrm{kDa}$. (B) EGF application to LN18 cells did not modify LC3B-II levels. (C) EGFR and Beclin-1 do not bind in LN18 cells. Immunoblotting of Co-IP reveals the pull-down of EGFR in the first lane, the pull-down of Beclin-1 in the second lane and the original lysate in the final lane. Beclin-1 antibody caused a smear in all Co-IP blots. This blurred spot was observed at a lower position compared with Beclin-1 in the blot. (D) EGFR and Beclin-1 do not interact following treatment with EGF. Cells were previously treated with EGF $(20 \mathrm{ng} / \mathrm{ml}, 30 \mathrm{~min})$ and Co-IP was performed by pull-down of Beclin-1. EGFR, epidermal growth factor receptor; LC3B-II, light chain 3 B-II; Co-IP, co-immunoprecipitation; EGF, epidermal growth factor.
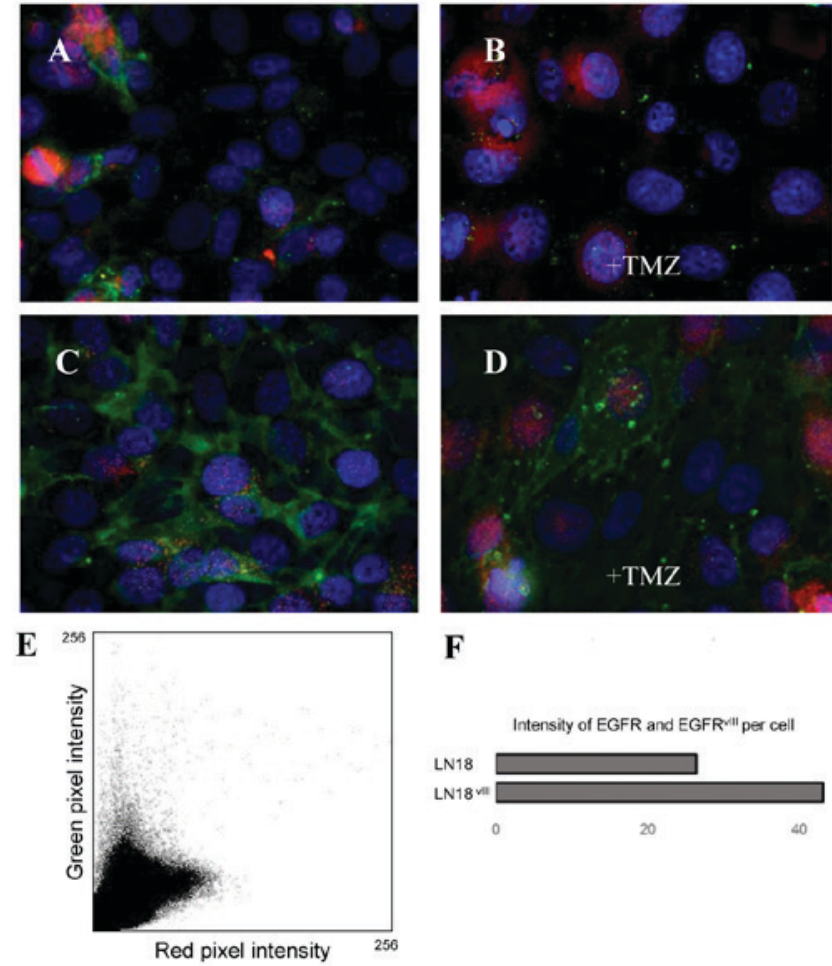

F

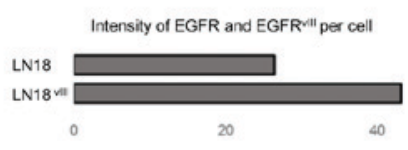

Figure 4. Immunofluorescence double-labeling of Beclin-1 (red) and EGFR (green). (A) GBM cell line LN18, (B) GBM cell line LN18+TMZ, (C) GBM cell line LN18 ${ }^{\mathrm{vIII}}$ and (D) GBM cell line LN18 ${ }^{\mathrm{vII}}+\mathrm{TMZ}$. Magnification, x63. Microphotographs reveal Beclin-1 protein expression at similar levels in the two cell lines, whereas LN18 $8^{\text {vIII }}$ expresses higher levels of EGFR. EGFR and Beclin-1 are not directly adjacent to one another. (E) Intensity of green and red pixels were identified per cell and analyzed for colocalization with the plugin 'colocalization colormap' in ImageJ. The scatterplot reflects the lack of correlation by the two separate groups of points. This was verified for each cell line and each condition. Pearson's correlation coefficient (pixel-by-pixel covariance): 0.39 (LN18), 0.00 (LN18+TMZ), 0.01 (LN18 ${ }^{\text {vIII })}$ and -0.06 (LN18 $\left.8^{\text {vIII }}+\mathrm{TMZ}\right)$. (F) Results of an analysis of 6 different immunofluorescence images of each cell line of one experiment. EGFR, epidermal growth factor receptor; LN18 ${ }^{\text {vIII }}$, constitutively active EGFR ${ }^{\text {vIII }}$ variant, TMZ, temozolomide. options (23). At present, the alkylating drug TMZ is used as the first-line treatment for primary GBM (1). The heterogeneous character of GBM cells complicates the evaluation of interference of TMZ with various cellular signaling pathways (24). However, the primary influences of TMZ on cellular signaling pathways require elucidation to analyze adverse side effects as well as potential accompanying therapeutic approaches. One affected pathway appears to be the catabolic process of autophagy (5). The conversion of LC3 has now become the most widely used method to monitor autophagy (8). An increase of LC3B-II, as presented in Fig. 1A, may be induced in primary and established GBM cell lines by autophagy regulation using chloroquine. This drug inhibits the fusion of lysosomes with autophagosomes, resulting in an accumulation of LC3B-II (10). Chloroquine has been used in several studies to suppress tumor growth, including in lung cancer cells (25). In GBM, the effects of chloroquine were promising and led to a significant extension of overall patient survival time (26).

Primary cell culture, which is an improved mimicry of relevant in vivo conditions, was compared to established GBM cell lines, as they are hypothesized to vary in dedifferentiation (27). The investigated primary cell lines pGBM T1 and pGBM T12 did not express MGMT, in contrast to LN18. The findings of the current study indicate that autophagy is increased in primary cell lines, predominantly in pGBM T1, by a lower concentration of TMZ and reduced treatment time compared with the established cell line LN18. The positive regulation of TMZ on autophagy may be interpreted as a response to adverse cellular conditions (5). The LN18 cells were not affected by low dose and short-term treatment with TMZ in the present study, which indicates TMZ-resistant characteristics. However due to adverse side effects, the high dose of TMZ required for autophagy induction in LN18 is not feasible for use in patients with resistant GBM. Taken together, this process of hindering cells from escaping adverse cellular 
conditions using chloroquine may be a promising addition to TMZ treatment.

Another established underlying mechanism to circumvent TMZ-induced damages is MGMT, which removes methylated DNA adducts (4). The expression of MGMT is a negative predictive factor for survival time in patients with GBM (28). In the current study, LN18 cells expressed MGMT, but the protein levels were markedly decreased by TMZ (Fig. 2). This indicates that the cellular supply of MGMT is exhausted when repairing methylated TMZ lesions, concordant with the findings of Wick et al (29). The primary cell lines pGBM T1 and pGBM T12 did not express MGMT, which may be interpreted as favorable regarding TMZ treatment.

The transmembrane signaling module EGFR serves a significant role in numerous cellular signaling pathways and its amplification or mutations are encountered in numerous cases of GBM, the most common of which is EGFR ${ }^{\text {vIII }}$ (15). Lacking its outer regulative portion, $\mathrm{EGFR}^{\mathrm{vIII}}$ provides constant signaling for the tumor cell (14). This signaling not only enhances phosphoinositide 3-kinase/protein kinase $\mathrm{B}$ activation, but also appears to possess divergent characteristics compared with wild-type EGFR signaling $(30,31)$. In the present study, the expression of the truncated VIII form appeared to be linked to higher resistance towards high-dose application of TMZ in LN18, as detected by immunofluorescence (Fig. 4). Notably, the co-expression of wild-type and truncated EGFR, as with LN18 ${ }^{\mathrm{vIII}}$, is hypothesized to result in an antagonistic connection between the two receptors (32). This may induce malignancy in GBM (32). However, the prognostic role of the truncated version of EGFR remains controversial. Shinojima et al (33) identified a poor prognostic outcome in patients with GBM due to EGFR $^{\mathrm{VIII}}$ in combination with EGFR amplification. By contrast, Montano et al (34) revealed a prolonged survival for patients with GBM expressing EGFR ${ }^{\mathrm{VIII}}$ (34). The altered signaling function of EGFR ${ }^{\mathrm{vIII}}$ and its implication in GBM growth and therapy resistance must be investigated in detail in the future.

Wei et al (13) suggested an important role for EGFR in the inhibition of autophagy initiation (13). This previous study described the phosphorylation of Beclin-1 by active EGFR in NSCLC cells (13). If EGFR in GBM cells also regulates Beclin-1, this is of note as EGFR is frequently amplified or mutated in GBM (35). In the current study, Beclin-1 was not phosphorylated by inactive or active EGFR independently of TMZ treatment in LN18 and LN18 ${ }^{\mathrm{vIII}}$. GBM cells did not exhibit modification of autophagy monitored by wild-type EGFR, application of EGF or EGFR $^{\mathrm{VIII}}$. Beclin-1 did not bind to EGFR or EGFR ${ }^{\mathrm{VIII}}$ in control cells or TMZ-treated cells in established and primary GBM. The interaction between Beclin-1 and EGFR remained unaffected by hypoxic conditions for $24 \mathrm{~h}$. Immunofluorescence revealed that Beclin-1 locations are not directly adjacent to EGFR locations in LN18 or LN18 ${ }^{\text {vIII }}$, which did not vary upon TMZ application. These data demonstrate that Beclin-1 does not interact directly with EGFR in the GBM cell lines used in the present study and suggests varied regulation, particularly for primary GBM culture. This is concordant with Zhu and Shah (36), suggesting other regulative pathways for autophagy than EGFR in GBM.

Further studies may focus on the interaction between inactive EGFR and autophagy. Tan et al (37) demonstrated that a knockdown of EGFR inhibited autophagy in different cell lines, but this study did not include brain tumor cells (37). However, these findings are in favor of an important stimulus of autophagy by inactive EGFR in certain tumor entities, and may be evaluated in GBM cells.

\section{Acknowledgements}

The authors would like to thank Dr Andreas Androutsellis-Theotokis (Carl Gustav Carus University, Dresden, Germany) for the cell line X01, Dr Daniela Schilling (Department of Radiooncology Technische Universität, Munich, Germany) for providing the hypoxia-incubating chamberand Mrs. Sandra Baur(Department of Neuropathology, Technische Universität, Munich, Germany) for technical support in the laboratory. This work was supported by the Deutsche Forschungsgemeinschaft (SFB 824: 'Imaging for the Selection, Monitoring, and Individualization of Cancer Therapies,' Project B6).

\section{References}

1. Koshy M, Villano JL, Dolecek TA, Howard A, Mahmood U, Chmura SJ, Weichselbaum RR and McCarthy BJ: Improved survival time trends for glioblastoma using the SEER 17 population-based registries. J Neurooncol 107: 207-212, 2012.

2. Stevens MF, Hickman JA, Stone R, Gibson NW, Baig GU, Lunt E and Newton CG: Antitumor imidazotetrazines. 1. Synthesis and chemistry of 8-carbamoyl-3-(2-chloroethyl)imidazo[5,1-d]-1,2,3, 5-tetrazin-4(3 H)-one, a novel broad-spectrum antitumor agent. J Med Chem 27: 196-201, 1984.

3. Agarwala SS and Kirkwood JM: Temozolomide a novel alkylating agent with activity in the central nervous system, may improve the treatment of advanced metastatic melanoma. Oncologist 5: 144-151, 2000.

4. Hegi ME, Diseren AC, Gorlia T, Hamou MF, de Tribolet N, Weller M, Kros JM, Hainfellner JA, Mason W, Mariani L, et al: MGMT gene silencing and benefit from temozolomide in glioblastoma. N Engl J Med 325: 997-1003, 2005.

5. Kanzawa T, Germano IM, Komata T, Ito H, Kondo Y and Kondo S: Role of autophagy in temozolomide-induced cytotoxicity for malignant glioma cells. Cell Death Differ 11: 448-457, 2004.

6. Natsumeda M, Aoki H, Miyahara H, Yajima N, Uzuka T, Toyoshima Y, Kakita A, Takahashi H and Fujii Y: Induction of autophagy in temozolomide treated malignant gliomas, Neuropathology 31: 486-493, 2011.

7. Yang Z and Klionsky DJ: Eaten alive: A history of macroautophagy. Nat Cell Biol 12: 814-822, 2010.

8. Mizushima N, Yoshimorim T and Levine B: Methods in mammalian autophagy research. Cell 140: 313-326, 2010.

9. Fujita N, Hayashi-Nishino M, Fukumoto H, Omori H, Yamamoto A, Noda T and Yoshimori T: An Atg4B mutant hampers the lipidation of LC3 paralogues and causes defects in autophagosome closure. Mol Biol Cell 19: 4651-4659, 2008.

10. Yoon YH, Cho KS, Hwang JJ, Lee SJ, Choi JA and Koh JY: Induction of lysosomal dilatation, arrested autophagy and cell death by chloroquine in cultured ARPE-19 cells. Invest Ophthalmol Vis Sci 51: 6030-6037, 2010.

11. Cheng Y, Ren X, Hait WN and Yang JM: Therapeutic targeting of autophagy in disease: Biology and pharmacology. Pharmacol Rev 65: 1162-1197, 2013.

12. Sinha S and Levine B: The autophagy effector Beclin 1: A novel BH3-only protein. Oncogene 27 (Suppl 1): S137-S148, 2008.

13. Wei Y, Zou Z, Becker N, Anderson M, Sumpter R, Xiao G, Kinch L, Koduru P, Christudass CS, Veltri RW, et al: EGFR-mediated Beclin 1 phosphorylation in autophagy suppression, tumor progression, and tumor chemoresistance. Cell 154: 1269-1284, 2013

14. Okamoto I, Kenyon LC, Emlet DR, Mori T, Sasaki J, Hirosako S, Ichikawa Y, Kishi H, Godwin AK, Yoshioka M, et al: Expression of constitutively activated EGFRvIII in non-small cell lung cancer. Cancer Sci 94: 50-56, 2003. 
15. Gan HK, Kaye AH and Luwor RB: The EGFRvIII variant in glioblastoma multiforme. J Clin Neurosci 16: 748-754, 2009.

16. Ishii N, Maier D, Merlo A, Tada M, Sawamura Y, Diserens AC and Van Meir EG: Frequent co-alterations of TP53, p16/CDKN2A p14ARF, PTEN tumor suppressor genes in human glioma cell lines. Brain Pathol 9: 469-479, 1999.

17. Schäfer A, Teufel J, Ringel F, Bettstetter M, Hoepner I, Rasper M, Gempt J, Koeritzer J, Schmidt-Graf F, Meyer B, et al: Aldehyde dehydrogenase 1A1-a new mediator of resistance to temozolomide in glioblastoma. Neuro Oncol 14: 1452-1464, 2012.

18. Berezowska S, Diermeier-Daucher S, Brockhoff G, Busch R, Duyster J, Grosu AL and Schlegel J: Effect of additional inhibition of human epidermal growth factor receptor 2 with the bispecific tyrosine kinase inhibitor AEE788 on the resistance to specific EGFR inhibition in glioma cells. Int J Mol Med 26: 713-721, 2010.

19. Ciechomska IA, Przanowski P, Jackl J, Wojtas B and Kaminska B BIX01294, an inhibitor of histone methyltransferase, induces autophagy-dependent differentiation of glioma stem-like cells Sci Rep 6: 38723, 2016

20. Rappl A, Piontek G and Schlegel J: EGFR-dependent migration of glial cells is mediated by reorganisation of N-cadherin. J Cell Sci 121: 4089-4097, 2008.

21. Bobola MS, Tseng SH, Blank A, Berger MS and Silber JR: Role of O6-methylguanine-DNA methyltransferase in resistance of human brain tumor cell lines to the clinically relevant methylating agents temozolomide and streptozotocin. Clin Cancer Res 2: 735-741, 1996.

22. Klionsky DJ, Abdelmohsen K, Abe A, Abedin MJ, Abeliovich H, Acevedo Arozena A, Adachi H, Adams CM, Adams PD, Adeli $\mathrm{K}$, et al: Guidelines for the use and interpretation of assays for monitoring autophagy (III edition). Autophagy 12: 1-222, 2016.

23. Weller M, Cloughesy T, Perry J and Wick W: Standards of care for treatment of recurrent glioblastoma-are we there yet? Neuro Oncol 15: 4-27, 2013

24. Soeda1 A, Hara A, Kunisada T, Yoshimura S, Iwama1 T and Park D: The evidence of glioblastoma heterogeneity. Sci Rep 5 7979, 2015

25. Fan C, Wang W, Zhao B, Zhang S and Miao J: Chloroquine inhibits cell growth and induces cell death in A549 lung cancer cells. Bioorg Med Chem 14: 3218-3222, 2006.
26. Briceño E, Calderon A and Sotelo J: Institutional experience with chloroquine as an adjuvant to the therapy for glioblastoma multiforme. Surg Neurol 67: 388-391, 2007.

27. Seidel S, Garvalov BK and Acker T: Isolation and culture of primary glioblastoma cells from human tumor specimens. Methods Mol Biol 1235: 263-275, 2015.

28. Thon N, Kreth S and Kreth FW: Personalized treatment strategies in glioblastoma: MGMT promoter methylation status. Onco Targets Ther 6: 1363-1372, 2013.

29. Wick W, Platten M and Weller M: New (alternative) temozolomide regimens for the treatment of glioma. Neuro Oncol 11: 69-79, 2009.

30. Bleeker FE, Molenaar RJ and Leenstra S: Recent advances in the molecular understanding of glioblastomas. J Neurooncol 108: $11-27,2012$

31. Eskilsson E, Røsland G, Talasila K, Jahedi R, Leiss L, Saed H, Keunen O, Foerster S, Euskirchen P, Hossain J, et al: Distinct EGFR signaling in Glioblastoma: Wild-type EGFR promotes invasion while EGFRvIII drives prototypical SFK c-SRC activation to foster angiogenesis. Neuro Oncol 16 (Suppl 5): v3, 2014

32. Li L, Puliyappadamba VT, Chakraborty S, Rehman A, Vemireddy V, Saha D, Souza RF, Hatanpaa KJ, Koduru P, Burma S, et al: EGFR wild type antagonizes EGFRvIII-mediated activation of Met in glioblastomas. Oncogene 34: 129-134, 2015.

33. Shinojima N, Tada K, Shiraishi S, Kamiryo T, Kochi M, Nakamura H, Makino K, Saya H, Hirano H, Kuratsu J, et al: Prognostic value of epidermal growth factor receptor in patients with glioblastoma multiforme. Cancer Res 63: 6962-6970, 2003.

34. Montano N, Cenci T, Martini M, D'Alessandris QG, Pelacchi F, Ricci-Vitiani L, Maira G, De Maria R, Larocca LM and Pallini R: Expression of EGFRvIII in glioblastoma: Prognostic significance revisited. Neoplasia 12: 1113-1121, 2011.

35. Padfield E, Ellis HP and Kurian KM: Current therapeutic advances targeting EGFR and EGFRvIII in glioblastoma. Front Oncol 5: 5, 2015 .

36. Zhu Y and Shah K: Multiple lesions in receptor tyrosine kinase pathway determine glioblastoma response to pan-ERBB inhibitor PF-00299804 and PI3K/mTOR dual inhibitor PF-05212384. Cancer Biol Ther 15: 815-822, 2014.

37. Tan X, Thapa N, Sun Y and Anderson RA: A kinase-independent role for EGF receptor in autophagy initiation. Cell 160: 145-160, 2015. 